\title{
La socialización vecinal, un requerimiento de integración entre los macroproyectos con los barrios tradicionales y parques históricos. Caso de estudio: El Parque Morelos y el barrio El Retiro, en Guadalajara, Jalisco.
}

\author{
Juan Christopher Alcaraz Padilla \\ Departamento de Técnicas y Construcción y representación arquitectónica, \\ Universidad de Guadalajara. Guadalajara, México \\ E-mail: ing.arq_csalcaraz@hotmail.com
}

\begin{abstract}
Resumen. Las ciudades están en la constante búsqueda de adaptarse a las versátiles necesidades. La expansión territorial de la urbe requiere de más espacios públicos y áreas verdes. Los lugares ya consolidados, como los circundantes al primer cuadro o centro histórico, se encuentran amenazados por proyectos que pretenden poner a la ciudad al nivel de las principales metrópolis. Los barrios tradicionales junto a sus espacios públicos, como parques y jardines, suelen ser parte primordial en los nuevos proyectos. Pero, en ocasiones su enfoque no beneficia al lugar sino lo transforma y perjudica su esencia tradicional.

El Parque Morelos, antigua Alameda de la ciudad de Guadalajara, es el primer espacio público de la ciudad. Alrededor de él, han acontecido una amplia gama de cambios urbanisticos. Su morfología y actividades no fueron la excepción, el lugar paso de ser parte del "paseo dominical" a un parque histórico en donde se han intentado implementar macro proyectos como el Proyecto Alameda y la Ciudad Creativa Digital. Ambas propuestas ponían al Parque Morelos y el vecino barrio de El Retiro como puntos principales. Sin embargo, al no tomar en cuenta a los habitantes del lugar, los dos planteamientos concluyeron en una afectación al tejido urbano.

La presente investigación se enfoca en puntualizar lo indispensable que es la socialización vecinal ante los macro proyectos. Al igual que exponer lo acontecido en el Barrio de El Retiro y el Parque Morelos con la implementación del Proyecto Alameda y la Ciudad Creativa Digital.
\end{abstract}

Palabras clave: parques históricos, barrios tradicionales, patrimonio urbano, patrimonio medioambiental.

La ciudad de Guadalajara Jalisco, el Parque Morelos y el barrio El Retiro.

La ciudad de Guadalajara con alrededor de $1.521,741$ habitantes $^{1}$ es, indiscutiblemente, mayor a la que albergó en su momento a las doce manzanas fundacionales establecidas en el Valle de Atemajac aproximadamente el 14 de febrero de 1542. La urbe actualmente está, rodeada por su área metropolitana (AMG) que consiste en los municipios de Zapopan,
Tlajomulco de Zúñiga, San Pedro Tlaquepaque, Tonalá, El Salto, Zapotlanejo, Ixtlahuacán de los Membrillos y Juanacatlán. Asimismo, al norte, se encuentra el borde natural, la Barranca de Huentitán. Los habitantes de Guadalajara representan un poco más del $30 \%$ del total de la AMG.

El municipio de Guadalajara ya no puede incrementar su extensión territorial por su localización geográfica al centro de la AMG y costado de su borde natural. Los nuevos

http://dx.doi.org/10.4995/ISUFh2019.2019.9970

(cc) EY-NC-ND 2019, Editorial Universitat Politècnica de València 
asentamientos y puntos de centralidad, mayormente, se sitúan en los municipios circunstantes ya que su superficie no ha sido totalmente consumida por la mancha urbana. La ciudad quedó en un punto donde las edificaciones que se proponen tendrían que emerger sobre un inmueble existente ya sea reutilizándolo o retirándolo. Por lo que, desde hace tiempo, ha comenzado un proceso de reacondicionamiento de ciertos sectores, principalmente los contiguos al primer cuadro de la ciudad.

En el centro histórico o primer cuadro ${ }^{2}$ de Guadalajara, se han llevado a cabo varios macro proyectos a lo largo del tiempo. Se entiende como macro proyecto, aquella obra que haya modificado la traza o morfología en una superficie urbana consolidada, especialmente aquellas dentro de contextos históricos. Las obras de gran impacto han sido el entubamiento del río Juan de Dios (borde natural y limítrofe del oriente y poniente de la ciudad), la rectificación de la Calzada Independencia (eje norte sur relativamente paralelo al río San Juan de Dios), el mercado Libertad, la Cruz de Plazas y la Plaza Tapatía ${ }^{3}$. Cada uno de estos macro proyectos requirió el retiro de edificaciones patrimoniales, corrección de vialidades $\mathrm{y}$ reubicación de habitantes causando polémicas por sus alcances e implicaciones. No obstante, esos cambios llevaron a la ciudad que hoy en día se percibe.

El centro histórico está rodeado por barrios tradicionales y un parque histórico. Estos lugares y junto a sus habitantes han tenido la fortuna de permanecer aun cuando el uso de suelo predominante es el comercio. Asimismo, han sobrellevado la inseguridad nocturna en ciertos corredores por lo desolado al concluir los horarios laborales. En lado nororiente, adyacente a la Calzada Independencia y colindantes entre sí se localizan el Parque Morelos y el barrio El Retiro.

El Parque Morelos, antigua Alameda de la ciudad, es considerado como histórico por su permanencia desde la segunda mitad del siglo XVIII hasta la actualidad. Su morfología, al igual que su nombre y actividades, cambiaron por el entubamiento del rio, la rectificación de la Calzada Independencia y el fin del paseo dominical. Las intervenciones han pasado por manos de arquitectos del regionalismo tapatío ${ }^{4}$, movimiento moderno y despachos contemporáneos. Al presente, el parque ha mantenido una serie de problemáticas sociales derivadas de prácticas incompatibles a la recreación y ocio que han llevado deterioro de puntos específicos del lugar y la imagen urbana de su contexto.

Aledaño al Parque Morelos, hacia el norte, se encuentra el barrio El Retiro ${ }^{5}$. El nombre del lugar surge por la distancia de su localización con respecto al primer cuadro ubicándose en la sección limítrofe del norte. El barrio inició como un lugar donde predominaban las tenerías, detalle que le dio un aspecto peculiar eidentidad. Sus habitantes provenían, generalmente, de un municipio del norte del Estado, Yahualica. Las tenerías fueron decreciendo durante la apertura de productos extranjeros al país en el siglo XX. Hoy en día, es un barrio donde predomina el uso habitacional rodeado por equipamientos públicos de alto impacto que conllevan a una alta cantidad de habitantes de otros puntos del AMG.

La cercanía del Parque Morelos con el Barrio El Retiro y la interacción entre ciertas patologías urbanas, los llevaron a ser candidatos del primer macro proyecto del siglo XXI en el centro histórico. La administración municipal y estatal estaba en la búsqueda del rescate y recuperación de la zona, aunado a la tendencia de marcar las acciones del gobierno en turno y la creciente necesidad de dar a conocer a Guadalajara como capital al nivel de los estándares de las mejores ciudades del mundo, buscaron la postulación para ser la sede de Los Juegos Panamericanos que se llevarían a cabo del 14 al 30 de octubre del año 2011.

El proyecto Alameda (las Villas Panamericanas) y su afectación al tejido urbano en el contexto del Parque Morelos y El Retiro

En mayo de 2006, se recibió el fallo a favor de la ciudad de Guadalajara como centro de la justa internacional. La elección no sólo implicaba el recibir a la comunidad extranjera participante, sino la construcción de la infraestructura necesaria para las competencias de las distintas disciplinas y el alojamiento temporal de los asistentes. A partir de la anuencia de los organizadores se inició la búsqueda de sitios idóneos para la construcción 
de los espacios que alojarían a los deportistas.

La primera propuesta fue en la localidad de Tesistán, municipio de Zapopan, Jalisco, que fue rechazada por el aumento en el precio del terreno. La segunda opción, nuevamente en el municipio de Zapopan, estaba por la zona de avenida Acueducto que, nuevamente, se denegó por el costo de los predios. Finalmente, se optó por buscar una alternativa que pudiera posteriormente comercializarse como vivienda de nivel medio en la municipalidad de Guadalajara. Es allí que comenzaron a buscar predios alrededor del Parque Morelos y el barrio El Retiro después de que el sector del Parque Agua Azul y la barranca de Huentitán fueran cancelados por el gobierno municipal ${ }^{6}$.

El programa de necesidades del conjunto requería de una intervención en el Parque Morelos y la construcción de 13 edificios proyectados por firmas locales, nacionales e internacionales bajo el nombre de El Proyecto Alameda. Durante el proceso se adquirieron predios con base en distintos tipos de acuerdos. Sin embargo, los habitantes de la colonia El Retiro, se manifestaron contra del macro proyecto por el impacto que causaría en la zona y las repercusiones en el tejido social, ante la adquisición y demolición de predios. Las inconformidades de los vecinos continuaron a lo largo de varios meses y, asociado a eso, la crisis económica del año 2009 disminuyó la factibilidad de los edificios ya que cada uno tenía sus particularidades específicas. Se optó por recurrir a un nuevo proyecto que conjuntara las necesidades de los trece edificios y redujera los costos de construcción al uniformizar la modulación. La propuesta era un edificio en forma de L que rodeaba el norte y poniente del Parque Morelos, convirtiéndose en un borde invasivo que separaba el Parque y el barrio.

La polémica y las contantes demandas llevaron a la cancelación definitiva del Proyectó Alameda el día 7 de diciembre de 2009. Finalmente, la construcción fue trasladada a las inmediaciones del Bajío en el municipio de Zapopan bajo el nombre de las Villas Panamericanas ${ }^{7}$. El proyecto Alameda dejó, en el Parque Morelos y El Retiro, un alto índice de huecos urbanos, mayor grado de deterioro, aumento de actividades ilícitas y una huella urbana irreversible donde se perdieron vecinos, comercios tradicionales de la zona (las neverías) y conjuntos con posible valor patrimonial ambiental.

\section{La Ciudad Creativa Digital}

Durante la administración federal del año 2012, Guadalajara fue nuevamente postulada como sede de otro macro proyecto, la Ciudad Creativa Digital (CCD) compitiendo con ciudades como Puebla y Monterrey. El conjunto de la CCD consiste en un grupo de industrias creativas sostenibles basado en la identidad cultural, artística y tecnológica del lugar propuesto ${ }^{8}$. La administración en turno, buscaba promover a Guadalajara como la capital de la innovación y vio en la CCD la posibilidad de consolidar la imagen anunciada para el municipio. En abril del año 2012 el gobierno estatal y municipal aprobó la propuesta de construir la CDD en los predios baldíos del cancelado Proyecto Alameda en las inmediaciones del Parque Morelos y el barrio El Retiro.

El desarrollo del plan maestro estuvo a cargo del Instituto Tecnológico de Massachusetts (MIT). En él se plasmó el contexto de la $\mathrm{CCD}$, su función en Guadalajara, el Parque Morelos como corazón del proyecto, diseño urbano, estilos de vida digital, sustentabilidad, infraestructura urbana, estrategia económica y diagnósticos del sitio en tres distintas fases. En teoría, el plan maestro cubría gran parte de los parámetros necesarios para su correcta función partiendo de los estudios socioeconómicos, históricos y etnográficos, que se realizaron dentro de las áreas de intervención. El plan maestro propusó una serie de clusters $^{9}$ con la finalidad de invularlos con el centro histórico y otras zonas de alto impacto. Los estudios socioeconomicos y etnograficos de la CCD arrojó que la zona de El Retiro era de alto grado de marginación.

La primera fase comenzó con la construcción de la torre denominada $\mathrm{BOSCH}^{10}$. Sin embargo, la inceridumbre sobre los alcances e impactos reales de la $\mathrm{CCD}$, aunado a la alta cantidad de errores que tenía el plan maestro donde las principales discrepancias se encontraban en la realidad del contexto del Parque Morelos, dieron pie a la anulación del planteamiento propuesto por el MIT. El edificio de la fase 1 
continúo con su desarrollo hasta su conclusión en el año 2018. Durante el periodo del 2017 al 2018 se implementaron estrategias que replantearían la CCD señalando los beneficios del proyecto en distintos sectores, los habitantes del lugar (CCD LAB) y comunidad interesada en el desarrollo y estudio de tecnologías digitales (CCD Circuitos).

Los habitantes del lugar no quedaron convencidos con los posibles factores positivos. Mientras, que la comunidad interesada en tener instalaciones que ayuden a su desarrollo profesional se mostraron a favor de su continuidad. En el año 2019 durante el evento Talent Land se dio la noticia, por parte del gobierno estatal, que la Ciudad Creativa Digital se retomaría en las inmediaciones del Parque Morelos y el barrio El Retiro. Hasta la fecha de investigación, el macro proyecto sigue en función, pero a un ritmo sosegado. Los habitantes de El Retiro continúan pendientes de los avances de la construcción. Pero, su punto de vista, que protege su barrio, ahora está contrapuesto con la respuesta favorable de los interesados en desarrollar sus habilidades creativas en el complejo (jóvenes de la edad de 20 a 35 años). En el proyecto Alameda, está totalmente claro que no se incluyeron a los actores sociales involucrados en el proyecto. Pero, en la CCD irealmente se ha socializado?

La socialización vecinal como requerimiento de integración

Toda construcción, sin importar la dimensión, tiene un impacto en el lugar y el contexto. Desde la conceptualización de un proyecto a menor escala, generalmente, se involucra al interesado en la toma de decisiones ya que él o ella van a disfrutar o sobrellevar el resultado final. Lo mismo se tendría que realizar en los macro proyectos que, por lo particular, son promovidos por las administraciones gubernamentales encargando los proyectos a despachos particulares. A partir de la asignación, los profesionistas deberían comenzar a involucrar a los principales actores sociales del lugar, vecinos y otros especialistas del ramo de las humanidades previo al anteproyecto.

La interacción entre los profesionistas y la sociedad brinda un ambiente de confianza y aceptación de las propuestas es por ello que es ineludible la socialización vecinal. James W. Vander Zanden (1995) define la socialización como "el proceso por el cual los individuos, en su interacción con otros, desarrollan las maneras de pensar, sentir y actuar que son esenciales para su participación eficaz en la sociedad". La socialización vecinal como estrategia de integración entre los principales involucrados y los profesionistas, tiene varios procesos que varían con respecto al impacto del macro proyecto. Sin embargo, hay tres pasos que se pueden implementar en el ámbito general.

-El acercamiento y exposición general del macro proyecto.

- Propuestas a partir de las necesidades particulares de los habitantes.

-Presentación de anteproyectos y retroalimentación.

-Exposición de proyecto final.

El primer punto, el acercamiento con los habitantes particulares, es la esencia principal de la socialización. De allí se establece si los alcances generales del proyecto corresponden a la problemática del lugar. La importancia de comparar los objetivos y la problemática es indicador de la factibilidad real del proyecto, ya que en ocasiones los proyectos son pertinentes, pero, no en el entorno urbano al que se propone implementar, espacialmente en barrios históricos o contextos patrimoniales. Las propuestas preliminares deben de surgir a partir de las mesas de trabajo y discusiones con los principales actores sociales. Los trabajos presentados se retroalimentaran con los puntos de vista de los involucrados y profesionistas internos o externos. El proyecto final debe de ser la consecuencia de un trabajo en conjunto, donde los habitantes, en general, estén conformes y se integre con el contexto urbano y medioambiental.

En al caso particular del Proyecto Alameda y la Ciudad Creativa Digital en relación al Parque Morelos y el barrio El Retiro, se partió de las necesidades particulares de una limitada cantidad de personas involucradas en la toma decisiones. El resultado fue una fragmentación en el tejido social y urbano como ha sucedido en otras ciudades ${ }^{11}$. El planteamiento de ambos macro proyectos no comenzó por resolver las problemáticas de lo particular. El supuesto 
beneficio a todos los habitantes es ambiguo porque los primeros afectados no pertenecen a esa mayoría. Tal vez si se comenzara por interpolar las necesidades de los vecinos y los proyectos de vivienda, en el caso del Proyecto Alameda, se podría haber construido edificaciones que no fueran invasivas y se integraran al contexto. En el caso de la CCD es distinto por la implementación de industrias y otras dinámicas urbanas que, hasta la fecha, no aclaran la compatibilidad con el barrio, el Parque Morelos y su contexto.

Los macro proyectos tuvieron impactos negativos, pero ayudaron a consolidar un barrio. Los vecinos formaron su propia asociación en defensa de su territorio y espacio público, el Parque Morelos. Asimismo, manifestaron su apego y pertenencia, en otras palabras entendieron el valor del reconocimiento patrimonial de su entorno ${ }^{12}$. Su presencia se ha hecho más notable desde que lograron la cancelación del Proyecto Alameda y el Plan Maestro de la CCD. Los habitantes de la zona únicamente solicitan que se respete su espacio, los involucren en la toma de decisiones y se socialicen los proyectos con la finalidad de buscar un verdadero beneficio a favor de los primeros involucrados.

El reto actual que tienen los vecinos del barrio es estar al pendiente de los avances de la CCD y la protección del patrimonio medioambiental del Parque Morelos, su arbolado y áreas verdes, porque en los últimos años ha ido desapareciendo paulatinamente. Su atención y prevención es contante hasta que el proceso de socialización por parte de la municipalidad sea obligatoria previo a cualquier intervención de su parte.

\section{Reflexiones finales}

La socialización vecinal debe de ser un requerimiento obligatorio previo a la implementación de macro proyectos, especialmente en barrios tradicionales $\mathrm{y}$ parques históricos. La finalidad tiene que ser la integración puntual de lo que existe con lo que se pretende insertar. Los profesionistas encargados de los proyectos, necesitan realizar procesos etnográficos en sitio, entrevistas, escuchar y observar las problemáticas reales del lugar. Los proyectos no pueden concebirse desde un escritorio, se requiere la recolección de información de campo proveniente del sitio y sus habitantes. Un macro proyecto no está ligado a un lugar en específico, este siempre se puede acondicionar a otra ubicación donde realmente se complemente su función con el contexto y, en realidad, beneficie a su sector específico.

Los macro proyectos descritos, el Proyecto Alameda y la Ciudad Creativa Digital, son muestras de una grave falta de vinculación entre los vecinos, el proyecto y la integración con el parque histórico. En relación a este espacio, el Parque Morelos, ha sido parte primordial de ambos casos de intervención. Actualmente cuenta con aproximadamente 50,000 metros cuadrados de superficie, y tiene un alto índice de patrimonio medioambiental (flora y fauna). Sin embargo se ha modificado indiscriminadamente por parte de las autoridades causando afectaciones serias. Parte de la socialización es entender, por parte de los involucrados en la toma de decisiones, el valor histórico, social y medioambiental del lugar, así como, las patologías y dinámicas urbanas regentes en la zona de intervención. El insertar arquitectura reciente como parte de un macro proyecto, no soluciona los problemas sociales del entorno. Al contrario, está expuesto a ser deteriorado a corto plazo y aumentar la problemática.

El ejercicio diario de la arquitectura y el urbanismo requiere de formar equipos de trabajo multidisciplinarios y terminar la tendencia de proponer todas las soluciones desde un escritorio y con un sólo criterio, el del proyectista. La ciudad es dinámica y cambia constantemente, al igual que sus necesidades. Pero, los verdaderos requerimientos se obtienen por medio de la investigación del lugar $\mathrm{y}$, en su momento, la socialización de las intervenciones. Este proceso debe ser el obligatorio antes de cualquier proyecto definitivo y autorización de la obra. Asimismo, vincularla con políticas públicas que beneficien a los principales involucrados en lo particular y sea ejemplo de una buena integración contextual en lo general, evitando una ciudad segmentada y con ambiguos tejidos sociales entre barrios, colonias, espacios públicos, 
parques y centros históricos.

\section{Notas}

1 La cantidad de habitantes fue consultada en el Instituto de Información Estadística y Geográfica (IIEG) con base en su censo poblacional del 1 de julio de 2017. Cabe destacar, que la cifra es únicamente de la ciudad de Guadalajara sin su área metropolitana decretada en el año 2015. Consulta, https://iieg.gob.mx/strategos/ alcanza-area-metropolitana-de-guadalajaralos-5-millones-de-habitantes/

2 Federico Gorio en su publicación "Critica dell' idea di "centro storico". Rassegna di architettura e urbanística. define al centro histórico como: "núcleo original de la ciudad, es decir, a la parte más antigua o vieja de ésta, y por años se careció de una unidad de medida temporal con la cual medir su antigüedad o su historicidad, y de un criterio con el cual definir e limite espacial que lo separa de aquello que no es considerado histórico"

3 Cabe mencionar que existieron una amplia cantidad de modificaciones alrededor del primer cuadro de la ciudad pero las antes señaladas fueron las de mayor impacto.

4 El regionalismo o escuela tapatía es la arquitectura producida en la primera mitad del siglo XX por egresados de la escuela Libre de Ingenieros de Jalisco como Luis Barragán, Pedro Castellanos, Ignacio Díaz Morales y el autor de la primera intervención del Parque Morelos como se conoce hoy en día, Rafael Urzúa Arias.

5 Luis Francisco Talavera Durón indica que la“división geográfica actual del gobierno municipal, es, al norte, las calles Tenerías y Monte Cáucaso; al oriente, la Calzada Independencia; al sur, la calle Juan Manuel; y al poniente, las calles Dr. Baeza Alzaga y Coronel Calderón [...] Los barrios aledaños a El Retiro son, por el norte, Alcalde Barranquitas; por el oriente, cruzando la Calzada Independencia, el barrio La Perla; por el sur y el poniente, el Centro y Centro Barranquitas."

6 Ramón Reyes Rodriguez en su estudio sobre las Villas Panamericanas señala que "algunas razones que justificaron la selección del lugar fue el régimen de propiedad de los terrenos, pues, de acuerdo a la información oficial, en el perímetro del parque mencionado, el $70 \%$ de la propiedad inmobiliaria pertenece al gobierno municipal, estatal y federal y el restante a propietarios privados."

7 A la fecha de la investigación, el conjunto se encuentra sin uso por anomalías en el uso de suelo, impacto ambiental y conflictos políticos.

8 Monica Solórzano Gil indica que el programa fue propuesto por la UNESCO en octubre de 2004 con el fin de " promover el desarrollo social y económico de las ciudades tanto en países industrializados como en desarrollo, poniendo un énfasis especial en la función que desempeñen la creatividad y las artes en el desarrollo de las comunidades y las economías locales."

9 Conjunto de empresas que desarrollan actividades dentro del mismo sector.

10 El nombre correspondia a la empresa transnacional que se instalaria en el inmueble.

11 Laura Isabel Romero Castillo señala que "las inversiones en megaproyectos urbanos han implicado mayor fragmentación del territorio y de los lazos sociales preexistentes en las zonas intervenidas. Esto ha generado un fuerte contraste con las zonas inmediatas, cuya población puede ser y ha sido desplazada por diversos medios a nombre del interés público mediante la expropiación y la copra de tierra por parte de los gobiernos ocales a precios bajos y por los sectores sociales de mayores ingresos que están en condiciones de acceder a un suelo revalorizado."

12 María Patricia Pensado Leglise define el reconociendo patrimonial como "el impulso de las prácticas sociales y culturales que puedan intervenir en las decisiones para solucionar los problemas urbanísticos y que, a la vez, fortalezcan el sentido de pertenencia a la localidad y a la identificación de su comunidad con el entorno urbano: algo que va mucho más allá de la pretensión de acogerse a las leyes del patrimonio para salvar o revitalizar determinados lugares públicos." 


\section{Referencias}

Gorio, Federico. 1980. Critica dell' idea di 'centro storico. Rassegna di architettura e urbanística. Italia: Univertiá degli studi de Roma.

Massachussets Institute of technology. 2012. Plan Maestro de Guadalajara - Ciudad Creativa Digital. Boston: Massachussets Institute of Technology

Medina Ortega, Marco Antonio. 2016. "Ciudad Creativa Digital y su impacto metropolitano". En Cuadernos Con-texto 5, Ciudad y procesos metropolitano, 25-36. Guadalajara: Universidad de Guadalajara.

Pensado Leglise, María Patricia. 2012. "El reconocimiento patrimonial como estrategia vecinal". En El espacio público en la ciudad contemporánea, 109-120. México: Universidad Iberoamericana de Puebla e Instituto Universitario de Urbanística de la Universidad de Valladolid.

Reyes Rodríguez, Ramón. 2011. “Juegos Panamericanos y transformación morfológica e identitaria: el caso de la Villa Panamericana en Guadalajara". En La Villa Panamericana en Guadalajara, una visión multidisciplinaria de las primeras intervenciones urbanas en torno al Parque Morelos, 14-34. Guadalajara: Universidad de Guadalajara.

Romero Castillo, Laura Isabel. 2007. "Políticas públicas y megaproyectos urbanos en ciudades de importancia en el ámbito regional". En Los problemas de los espacios habitados y el medio ambiente. Perspectivas desde las ciencias y artes para el diseño, 43-62. México: Universidad Autónoma Metropolitana de México unidad Xochimilco.

Solórzano Gil, Mónica. 2015. Ciudades creativas y patrimonio cultural: nuevos escenarios para la conservación del patrimonio en México. Guadalajara: Instituto Tecnológico de Estudios Superiores de Occidente.

Talavera Durón, Luis Francisco. 2009. Los mil y un retiros en torno al Parque Morelos. La construcción social de un territorio. Guadalajara: H. Ayuntamiento de Guadalajara.
Vander Zanden, James W. 1995. Manual de Psicología Social. Barcelona: Editorial Paidós Ibérica. 